Article

\title{
Hybrid Grating in Reduced-Diameter Fiber for Temperature-Calibrated High-Sensitivity Refractive Index Sensing
}

\author{
Biqiang Jiang ${ }^{1} * \mathbb{C}^{\mathbb{D}}$, Zhen Hao ${ }^{1}$, Dingyi Feng ${ }^{1}$, Kaiming Zhou ${ }^{2}$, Lin Zhang ${ }^{2}$ and Jianlin Zhao ${ }^{1, *}$ \\ 1 MOE Key Laboratory of Material Physics and Chemistry Under Extraordinary Conditions and Shaanxi Key \\ Laboratory of Optical Information Technology, School of Science, Northwestern Polytechnical University, \\ Xi'an 710072, China; zhenhao@mail.nwpu.edu.cn (Z.H.); fengdingyi@nwpu.edu.cn (D.F.) \\ 2 Aston Institute of Photonic Technologies, Aston University, Birmingham B4 7ET, UK; \\ k.zhou@aston.ac.uk (K.Z.); l.zhang@aston.ac.uk (L.Z.) \\ * Correspondence: bqjiang@nwpu.edu.cn (B.J.); jlzhao@nwpu.edu.cn (J.Z.); Tel.: +86-29-8843-1663 (J.Z.)
}

Received: 9 April 2019; Accepted: 6 May 2019; Published: 10 May 2019

\begin{abstract}
We propose and experimentally demonstrate a hybrid grating, in which an excessively tilted fiber grating (Ex-TFG) and a fiber Bragg grating (FBG) were co-inscribed in a reduced-diameter fiber (RDF). The hybrid grating showed strong resonances due to coupling among core mode and a set of polarization-dependent cladding modes. This coupling showed enhanced evanescent fields by the reduced cladding size, thus allowing stronger interaction with the surrounding medium. Moreover, the FBG's Bragg resonance confined by the thick cladding was exempt from the change of the surrounding medium's refractive index (RI), and then the FBG can work as a temperature compensator. As a result, the Ex-TFG in RDF promised a highly sensitive RI measurement, with a sensitivity up to $\sim 1224 \mathrm{~nm} / \mathrm{RIU}$ near the RI of 1.38. Through simultaneous measurement of temperature and RI, the temperature dependence of water's RI is then determined. Therefore, the proposed hybrid grating with a spectrum of multi-peaks embedded with a sharp Bragg resonance is a promising alternative for the simultaneous measurement of multi-parameters for many RI-based sensing applications.
\end{abstract}

Keywords: fiber Bragg grating; tilted fiber grating; refractive index sensing; temperature dependence of the refractive index of water

\section{Introduction}

Refractive index (RI) measurement has an enormously broad range of applications, from biochemical industry to environmental monitoring and life science, where the sensing of many parameters can be converted to the RI measurement [1]. Compared with Abbe refractometers based on Snell's law and measurement of the critical angle of total reflection, optical fiber-based RI sensors have many unique advantages, for example their compact size, remote and distributed interrogation, and high sensitivity. A number of attempts employing fiber gratings and interferometers have been made to improve the RI sensitivity for many RI-based sensing applications [2-9]. Among these, long-period gratings (LPGs) and tilted fiber Bragg gratings (TFBGs) with abundant cladding mode resonances have demonstrated excellent performance in highly sensitive RI measurement by using several distinct demodulation techniques [10-14]. In order to further enhance the sensitivity and enlarge the sensing range, a few important approaches have been employed, i.e., increasing the tilted angle, reducing core or cladding's size, and coating functional materials onto the TFBGs. For instance, increasing the tilted angle and reducing the fiber diameter can shift the TFBG's cladding mode towards a shorter wavelength and enhance the evanescent field for a larger RI measurement range and higher 
sensitivity [15-18]. Similarly, coating TFBGs with noble metals to excite surface plasmon resonance (SPR) can effectively improve the RI sensitivity for biochemical and gas sensing [2,19-24]. Coating nanomaterials with high-complex RI can extend the measurement range, especially in the low-RI region [25-28].

Another special kind of so-called excessively tilted fiber grating (Ex-TFG) is characterized by a large tilted angle usually $>65^{\circ}$ and a set of polarization-dependent cladding mode resonances. This Ex-TFG is recognized for the highly sensitive nature of the light that interacts with the surrounding medium [29,30]. They have been developed and applied in the sensing of twist [31], refractive index [32], liquid-level [33], and biochemical parameters [34,35]. However, in these sensing applications using an Ex-TFG, the temperature cross-talk is a critical issue because there is no Bragg resonance to serve as a temperature reference like that of a TFG with small tilted angles.

In this work, we present a hybrid grating to achieve the high-sensitivity RI sensing with temperature calibration. An Ex-TFG and an FBG were UV-inscribed in the same segment of reduced-diameter fiber (RDF), showing a wide and multi-notch spectrum together with a sharp Bragg resonance. The use of an RDF for the Ex-TFG enhanced the accessible evanescent field for interacting with an external medium. The mode coupling of the FBG only occurred in the fiber core, with the light field was isolated from the surrounding by the cladding, and was thus exempt from disturbance by the change of surrounding medium's RI. As a result, the Ex-TFG in RDF provided a highly sensitive RI measurement, and the FBG was used as a temperature compensator. In the sensing experiments of temperature and RI, the hybrid grating demonstrated enhanced sensing ability and was able to be employed to examine the temperature dependence of water's RI. Therefore, the proposed hybrid grating promises applications in simultaneous measurement of multi-parameters.

\section{Principle and Device Fabrication}

Figure 1a depicts a schematic diagram of the proposed hybrid grating structure, containing an Ex-TFG and an FBG. It is well known that in a standard FBG, the only strong coupling permitted occurs between the forward- and backward-propagating core modes, for which the light is confined to near the fiber axis and is isolated from the surroundings by the relative thick cladding [36]. However, for the Ex-TFG with largely tilted fringes, the forward-propagating core mode was coupled into forward-propagating cladding modes according to the phase-match condition $[37,38]$. Hence, a Bragg resonance was embedded in a series of cladding mode resonances at discrete wavelengths, so that each cladding mode resonance had two orthogonal TE and TM polarization states. The Bragg wavelength $\lambda_{\mathrm{B}}$ of the FBG and the resonant wavelength $\lambda_{i, \text { clad }}^{m}$ of the $m$ th cladding for the Ex-TFG, are determined by the phase-match condition and can be expressed as follows

$$
\begin{gathered}
\lambda_{\mathrm{B}}=2 n_{\text {eff,core }} \Lambda_{\mathrm{B}}, \\
\lambda_{i, \text { clad }}^{m}=\left[n_{\text {eff,core }}-n_{i, \text { eff,clad }}^{m}\right] \Lambda_{\mathrm{G}} / \cos \theta .
\end{gathered}
$$

where, $i$ represents TE or TM modes, $n_{\text {eff,core }}$ and $n_{i, \text { eff,clad }}^{m}$ are the effective indices of the core mode and the $m$ th cladding mode, respectively, $\Lambda_{\mathrm{B}}$ and $\Lambda_{\mathrm{G}}$ are the grating period of the FBG and nominal period of the Ex-TFG, respectively, and $\theta$ is the tilt angle. The resonant wavelengths of the TE and TM cladding modes of Ex-TFG with the same order could have separation depending on the difference of their effective indices. From Equations (1) and (2), the resonant wavelength $\lambda_{i, \text { clad }}^{m}$ of the Ex-TFG is affected by the surrounding medium's RI. However, any changes of temperature and strain to the hybrid grating will produce shift in both $\lambda_{\mathrm{B}}$ and $\lambda_{i, \text { clad }}^{m}$. 
(a)

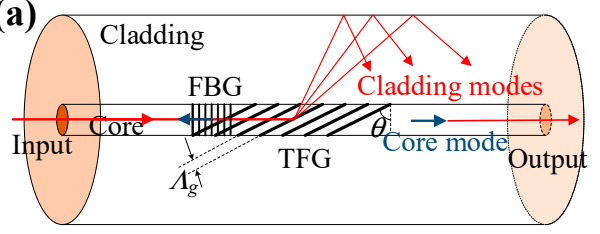

(b)

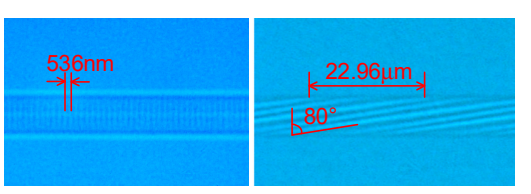

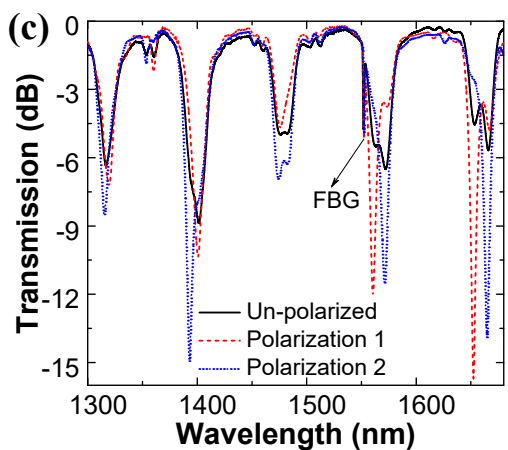

Figure 1. (a) Schematic model of the hybrid grating with an FBG and an excessively tilted fiber grating (Ex-TFG) in the reduced-diameter fiber (RDF); (b) optical microscopic images of the FBG and Ex-TFG fringes, and (c) transmission spectrum of the hybrid grating.

In the device fabrication, the $\mathrm{B} / \mathrm{Ge}$ co-doped silica RDF with $6.8 / 80 \mu \mathrm{m}$ core and cladding diameters was initially hydrogen loaded at $80^{\circ} \mathrm{C}$ and 150 bar pressure for $48 \mathrm{~h}$ to enhance its photosensitivity. An FBG was fabricated in a hydrogenated RDF by scanning a phase-mask with a period of $1064 \mathrm{~nm}$ using a frequency doubled continuous wave argon-ion laser. By switching to an amplitude-mask and rotating it by an angle with respect to the fiber axis, the Ex-TFG was then inscribed on top of the FBG. The hybrid grating with the FBG and Ex-TFG was annealed at $80^{\circ} \mathrm{C}$ for $48 \mathrm{~h}$ to release the residual hydrogen and stabilize the grating structure [33]. The lengths of FBG and Ex-TFG are $L_{F B G}$ $=1 \mathrm{~mm}$ and $L_{\mathrm{Ex}-\mathrm{TFG}}=5 \mathrm{~mm}$, respectively. Inspected with a high-resolution microscope, the optical microscopic images shown in Figure $1 \mathrm{~b}$ demonstrate that the periods of the FBG and Ex-TFG are 536 $\mathrm{nm}$ and $22.96 \mu \mathrm{m}$, respectively, and the tilted angle of Ex-TFG is around $80^{\circ}$.

The spectrum and sensing performance of the hybrid grating were examined by the experimental setup shown in Figure 2, which includes a supercontinuum (SuperK) light source, an in-fiber linear polarizer, polarization controller (PC), and an optical spectrum analyzer (OSA). Launched from the SuperK source, light was polarized and adjusted by the polarizer and the controller, respectively. The transmission spectrum was monitored by the OSA with a wavelength resolution of $0.02 \mathrm{~nm}$. Figure $1 \mathrm{c}$ shows the full spectrum of the hybrid grating in the range of 1300-1680 nm, including a sharp Bragg resonance dip at the FBG and a set of cladding mode resonance dips at the Ex-TFG in two orthogonal polarization states. For a specific order of cladding mode such as the peak at around $1560 \mathrm{~nm}$, the separation of dual-peak is approximately $9.50 \mathrm{~nm}$, which is larger than that of the Ex-TFG in standard single-mode fiber (SMF) [35,38]. Since the mode coupling of the FBG only occurs in the fiber core, and the light field is isolated from the surrounding by a relatively thick cladding, it is insensitive to surrounding RI. As a result, the Bragg resonance gives a reference of the variation of TFG cladding mode resonances with the change of environmental parameters.

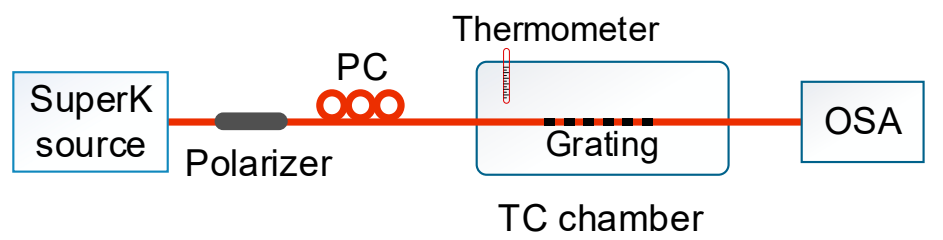

Figure 2. Experimental setup for testing spectrum properties of the hybrid grating. PC: Polarization controller; TC chamber: Temperature-control chamber; OSA: Optical spectral analyzer.

\section{Sensing Performance Tests and Discussions}

\subsection{Temperature Response}

To examine the temperature response, the hybrid grating was placed in a temperature-controllable chamber with a calibrated temperature probe. As the temperature increased, the grating period and RI 
modulation changed due to thermal expansion and thermal-optic effects, resulting in a shift in the wavelengths of Bragg resonance and cladding mode resonances. The hybrid grating was heated from $25-80^{\circ} \mathrm{C}$, and its spectrum was recorded with a step of $5^{\circ} \mathrm{C}$. The results are shown in Figure 3a. Clearly, the spectrum showed a global red-shift with the temperature increasing. In a detailed examination, we selected the cladding mode with resonance wavelength at $1561.4 \mathrm{~nm}$ and the Bragg resonance to investigate the temperature response, as shown in Figure 3b. The results show that the Bragg and cladding mode resonances shift linearly with the temperature, with response coefficients of $9.68 \mathrm{pm} /{ }^{\circ} \mathrm{C}$ and $39.65 \mathrm{pm} /{ }^{\circ} \mathrm{C}$, respectively.
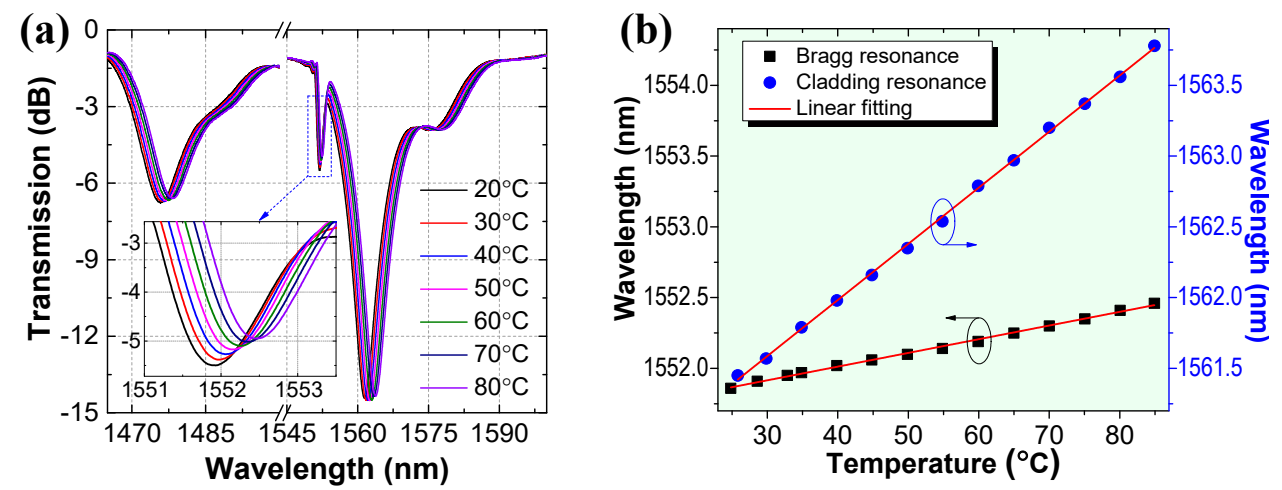

Figure 3. (a) Spectral evolution with the temperature; (b) temperature responses of Bragg resonance and cladding mode resonance of the hybrid grating.

\subsection{Refractive Index Response}

We examined the RI response using the experimental setup as described in [35]. The polarized light was coupled into the hybrid grating via the polarizer and the polarization controller, and the transmission signal was monitored and recorded by the OSA. A series of RI matching liquids (from Cargille Labs) ranging from 1.300-1.400 with an interval of 0.01 (with a maximum error of 0.0002 standardized at $589.3 \mathrm{~nm}$ and $25^{\circ} \mathrm{C}$ ) were used to test the device. The measured spectra of the hybrid grating for various RIs are depicted in Figure 4a. A significant red-shift in the cladding mode resonance and an increase in the coupling intensity was observed, especially in the shorter wavelength region. That is because the surrounding medium's RI modulates the effective index and coupling coefficient of the cladding mode.
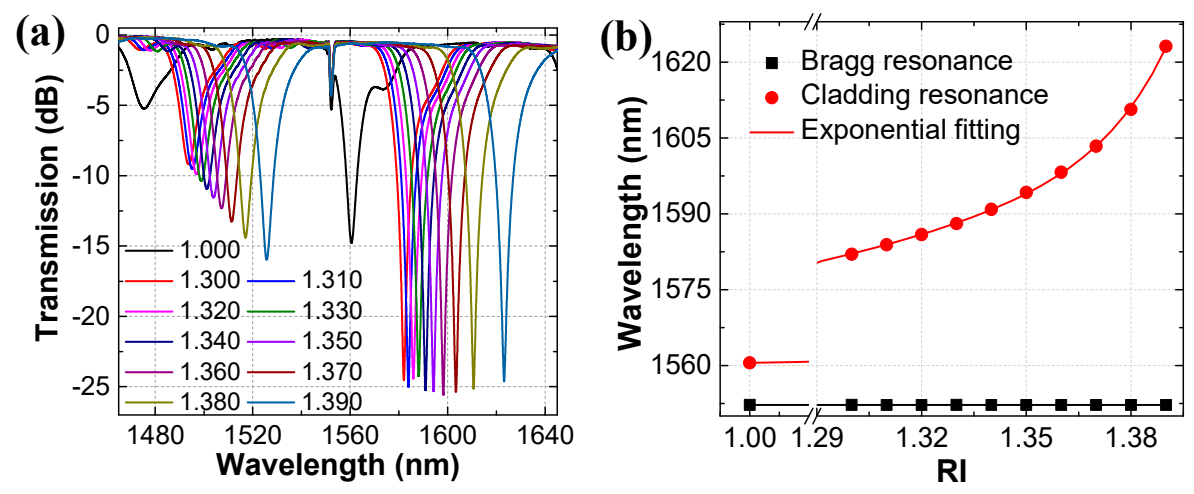

Figure 4. (a) Spectral evolution with the refractive index (RI) increasing; (b) RI responses of Bragg resonance and cladding mode resonances of the hybrid grating.

Figure $4 \mathrm{~b}$ plots the resonant wavelengths versus the RI value. The resonant wavelength demonstrated an exponential red-shift trend with increasing RI in the range of 1.300-1.400. According to the exponential fitting result $\lambda_{\text {Ex-TFG }}=1.01 \exp \left(n_{\mathrm{RI}} / 0.078\right)+1561.34(\mathrm{~nm})$, the calculated RI sensitivity was up to $~ 1224 \mathrm{~nm} / \mathrm{RIU}$ (RIU stands for "refractive index unit") near the RI of 1.38. The comparison 
results of sensitivity between different fiber grating-based RI sensors are shown in Table 1 . The sensitivity of the proposed sensor was much higher than that of most grating-based refractometers. For instance, the sensitivity of an LPG with a small period was around $312.5 \mathrm{~nm} / \mathrm{RIU}$ [8] and can be enhanced to $1005 \mathrm{~nm} / \mathrm{RIU}$ by inscribing the LPG in a hollow eccentric fiber [9]. The TFBGs with a small tilted angle in different fibers had the sensitivity of 500-574.2 nm/RIU by tracking the cut-off wavelength $[13,14,16,39]$. The sensitivity of SPR-assisted TFBGs with noble metals or nanowires coating reached to $510.5-651 \mathrm{~nm} / \mathrm{RIU}$ by monitoring the SPR resonance wavelength. The TFBG with high RI film (ZnO) had a sensitivity of $210 \mathrm{~nm} / \mathrm{RIU}$ at the RI of 1.39. The TFGs with large tilted angle showed a sensitivity of $\sim 340 \mathrm{~nm} / \mathrm{RIU}$ by tracking the resonance wavelength of the cladding mode. For the proposed sensor, the use of an RDF for the Ex-TFG enhanced the penetration of the evanescent wave of the cladding modes into the external medium and increased the RI dependence of waveguide dispersion, which is critical in determining the sensitivity [32,40,41]. Meanwhile, as the RI changed, the Bragg wavelength of FBG remained unchanged, which provides the feasibility for simultaneous measurement of temperature and RI.

Table 1. Comparison of sensitivity between fiber grating-based RI sensors.

\begin{tabular}{ccc}
\hline Sensing Structure & Sensitivity & RI Region \\
\hline LPG with a small period [8] & $312.5 \mathrm{~nm} / \mathrm{RIU}$ & $1.315-1.395$ \\
LPG in hollow eccentric fiber [9] & $1005 \mathrm{~nm} / \mathrm{RIU}$ & $1.422-1.441$ \\
$10^{\circ}$ TFBG [13] & $\sim 556 \mathrm{~nm} / \mathrm{RIU}$ & $1.315-1.360$ \\
Tilted moiré FBG [14] & $-574.2 \mathrm{~nm} / \mathrm{RIU}$ & $1.390-1.445$ \\
Multi-angle TFBG [16] & $500 \mathrm{~nm} / \mathrm{RIU}$ & $1.15-1.45$ \\
Multi-mode TFBG [39] & $\sim 570 \mathrm{~nm} / \mathrm{RIU}$ & $1.41-1.44$ \\
Gold-coated TFBG [22] & $510.5 \mathrm{~nm} / \mathrm{RIU}$ & $1.335-1.432$ \\
Gold-coated TFBG in polymer fiber [23] & $\sim 550 \mathrm{~nm} / \mathrm{RIU}$ & $1.408-1.428$ \\
Silver nanowires-coated TFBG [24] & $\sim 651 \mathrm{~nm} / \mathrm{RIU}$ & $1.330-1.347$ \\
ZnO-coated TFBG [26] & $210 \mathrm{~nm} / \mathrm{RIU}$ & $\sim 1.39$ \\
$>80^{\circ}$ Ex-TFG [29,33] & $340 \mathrm{~nm} / \mathrm{RIU}$ & $\sim 1.33$ \\
Hybrid grating in RDF (This work) & $\sim 1224 \mathrm{~nm} / \mathrm{RIU}$ & $\sim 1.38$ \\
\hline
\end{tabular}

\subsection{Temperature Dependence of Refractive Index of Water}

It is well known that the RI of transparent liquids and solids are functions of temperature. Taking a direct determination of the temperature dependence of water's RI as an example, the ability of simultaneous measurement of the RI and temperature may be verified by using the hybrid grating in an RDF. Based on the water-bath method, the hybrid grating was immersed in the distilled and deionized water, and the temperature was controlled with a heater and monitored with a thermometer. When the water was heated, there were two effects occurring, including the thermo-optic and thermal expansion effects of the hybrid grating itself, and the RI change of the water. Figure 5a shows the experiment results. The Bragg resonance had the same temperature response coefficient as that of air because it was not affected by the surrounding RI change. However, it is worth noting that the resonant wavelength of the cladding mode did not monotonically shift with the increase in temperature. We can employ the temperature calibration relationship of Bragg and cladding mode resonances to eliminate the inherent thermo-optic and thermal expansion effects of the grating induced by the temperature change. The result is shown in Figure 5b. It can be seen that the wavelength shift of the cladding mode after calibration was much larger than that before calibration. So, with the temperature rising from $25-80{ }^{\circ} \mathrm{C}$, the wavelength shift above $2.5 \mathrm{~nm}$ was caused by the dominated negative $\mathrm{d} n / \mathrm{d} T$ of water, showing a monotonic change. 
(a)
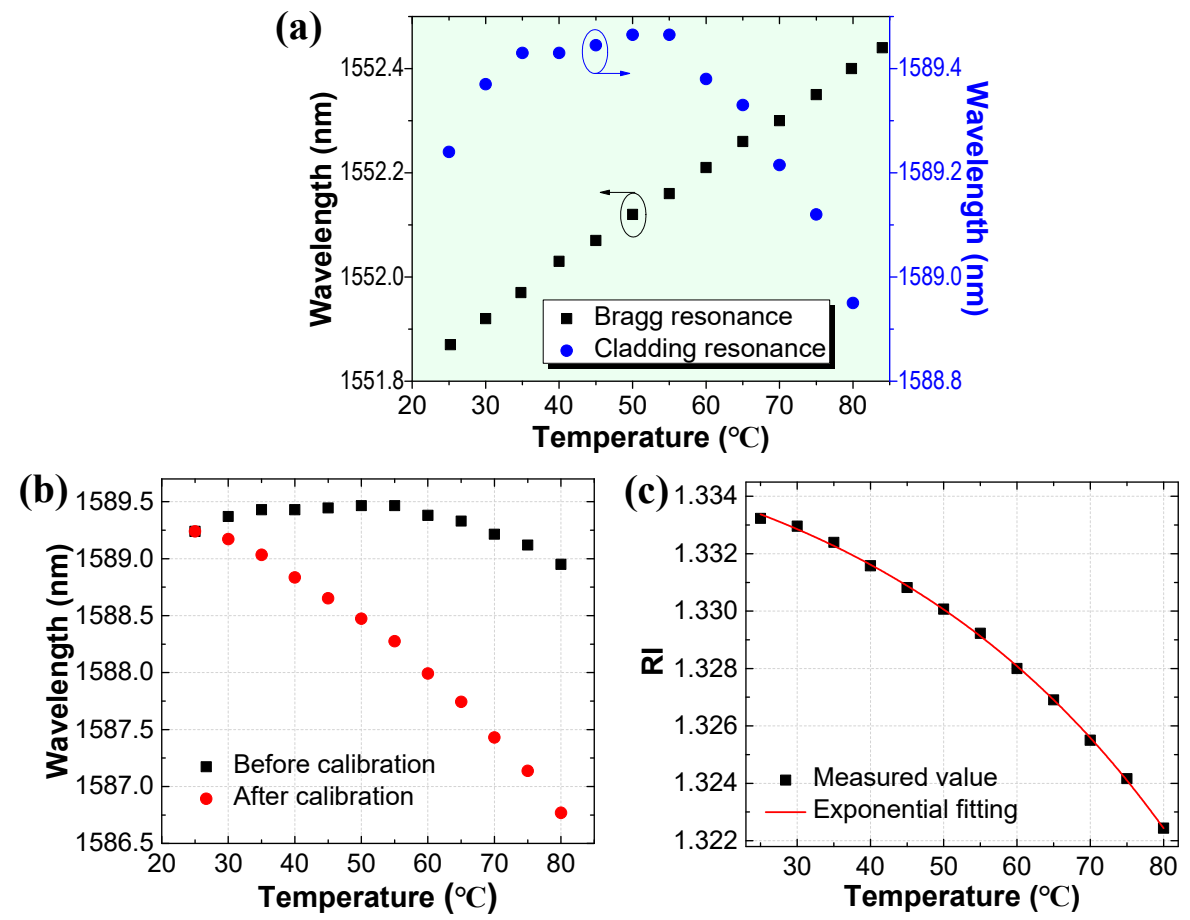

Figure 5. As the temperature increased, (a) original wavelength shifts of the Bragg and cladding mode resonances when the hybrid grating is in water; $(\mathbf{b})$ the wavelength shifts of the cladding mode resonance before and after temperature calibration; (c) variation of the calculated RI with the temperature.

As mentioned before, each wavelength of the cladding mode corresponds to a specific RI value of surrounding medium after the removal of inherent thermal effects of the grating. And then, we can calculate the corresponding RI values at each temperature by the calibration results plotted in Figure $4 \mathrm{~b}$. The obtained RI decreased nonlinearly with increasing temperature, and the fitting curve shows a nonlinear RI change of water in the temperature range of $25^{\circ} \mathrm{C} \sim 80^{\circ} \mathrm{C}$, which is very similar to the relationships from the previous reports [42-44]. The exponential relationship of water RI versus temperature in Celsius was $n=-0.00233 \cdot \exp (T / 42.74454)+1.33755$, with the R-square of 0.99945 .

In view of the above results, the contributions of the hybrid structure composed of two gratings in RDF are as follows: a) The sharp Bragg resonance of the FBG produced a stable temperature response that was exempt from surrounding RI change; b) The Ex-TFG provided an accessible evanescent field to enhance the interaction between light and the surrounding medium; and c) The use of RDF can help to enhance this effect and then the RI sensitivity. Meanwhile, although the temperature dependence of water RI as an example was investigated, to demonstrate simultaneous sensing performance of dual-parameter, the actual applications could be extended to the dynamic and static high-sensitivity measurement of RI and temperature. And also, the measurement accuracy could be further improved by using a set of standard RIs of to-be-measured liquid to strictly pre-calibrate the spectra of the hybrid grating at different RIs and temperature.

\section{Conclusions}

We have implemented a hybrid grating with an FBG and Ex-TFG in a special fiber with a small diameter to achieve the temperature-calibrated high-sensitivity RI sensing. The hybrid grating in the RDF promised the strong core-to-core and core-to-cladding mode coupling. It showed a high RI sensitivity with its enhanced evanescent field for interacting with the surrounding medium, and was also capable of measuring RI at different temperatures. The device demonstrated an RI sensitivity up to $\sim 1224 \mathrm{~nm} / \mathrm{RIU}$ at the RI of 1.38 and the thermal sensitivities of $9.68 \mathrm{pm} /{ }^{\circ} \mathrm{C}$ and $39.65 \mathrm{pm} /{ }^{\circ} \mathrm{C}$ for its Bragg and cladding mode resonances, respectively. By exploiting the RI-insensitive property of Bragg resonance, the temperature and RI were be monitored simultaneously, and the measurement of RI of 
water was calibrated at different temperatures. Therefore, the proposed hybrid grating in RDF with a compact and integrated structure provides a new approach to enhancement of the RI sensitivity and simultaneous measurement of multi-parameter for many RI-based sensing applications.

Author Contributions: Conceptualization, B.J. and L.Z.; methodology, K.Z.; validation, B.J. and Z.H.; formal analysis, D.F.; investigation, B.J. and Z.H.; writing-original draft preparation, B.J.; writing-review and editing, D.F. and K.Z.; supervision, L.Z and J.Z.; project administration, L.Z. and J.Z.; funding acquisition, B.J. and L.Z.

Funding: This research was funded by the National Natural Science Foundation of China (Grant Nos. 61775182, 61505165 and 61705185), the Natural Science Basic Research Plan in Shaanxi Province of China (Grant Nos. 2019JM-330 and 2018JQ6101), and the Marie Skłodowska-Curie Individual Fellowships in the European Union's Horizon 2020 Research and Innovation Programme (Grant No. 660648).

Acknowledgments: This research was supported by the Fundamental Research Funds for the Central Universities (No. 3102019PB005).

Conflicts of Interest: The authors declare no conflict of interest.

\section{References}

1. Zhou, W.; Zhou, Y.; Albert, J. A true fiber optic refractometer. Laser Photonics Rev. 2017, 11, 1600157. [CrossRef]

2. Caucheteur, C.; Guo, T.; Liu, F.; Guan, B.-O.; Albert, J. Ultrasensitive plasmonic sensing in air using optical fibre spectral combs. Nat. Commun. 2016, 7, 13371. [CrossRef]

3. Jin, W.; Cao, Y.; Yang, F.; Ho, H.L. Ultra-sensitive all-fibre photothermal spectroscopy with large dynamic range. Nat. Commun. 2015, 6, 6767. [CrossRef]

4. Shao, L.-Y.; Yin, M.-J.; Tam, H.-Y.; Albert, J. Fiber optic pH sensor with self-assembled polymer multilayer nanocoatings. Sensors 2013, 13, 1425-1434. [CrossRef]

5. Wang, Y.; Shen, C.; Lou, W.; Shentu, F.; Zhong, C.; Dong, X.; Tong, L. Fiber optic relative humidity sensor based on the tilted fiber Bragg grating coated with graphene oxide. Appl. Phys. Lett. 2016, 109, 031107. [CrossRef]

6. Li, Z.; Liao, C.; Chen, D.; Song, J.; Jin, W.; Peng, G.-D.; Zhu, F.; Wang, Y.; He, J.; Wang, Y. Label-free detection of bovine serum albumin based on an in-fiber Mach-Zehnder interferometric biosensor. Opt. Express 2017, 25, 17105-17113. [CrossRef]

7. Andrews, N.L.P.; Ross, R.; Munzke, D.; van Hoorn, C.; Brzezinski, A.; Barnes, J.A.; Reich, O.; Loock, H.-P. In-fiber Mach-Zehnder interferometer for gas refractive index measurements based on a hollow-core photonic crystal fiber. Opt. Express 2016, 24, 14086-14099. [CrossRef]

8. Shen, F.; Wang, C.; Sun, Z.; Zhou, K.; Zhang, L.; Shu, X. Small-period long-period fiber grating with improved refractive index sensitivity and dual-parameter sensing ability. Opt. Lett. 2017, 42, 199-202. [CrossRef]

9. Guan, C.; Tian, X.; Li, S.; Zhong, X.; Shi, J.; Yuan, L. Long period fiber grating and high sensitivity refractive index sensor based on hollow eccentric optical fiber. Sens. Actuators B Chem. 2013, 188, 768-771. [CrossRef]

10. Jiang, B.; Zhou, K.; Wang, C.; Zhao, Y.; Zhao, J.; Zhang, L. Temperature-calibrated high-precision refractometer using a tilted fiber Bragg grating. Opt. Express 2017, 25, 25910-25918. [CrossRef]

11. Guo, T.; Liu, F.; Guan, B.-O.; Albert, J. [INVITED] Tilted fiber grating mechanical and biochemical sensors. Opt. Laser Technol. 2016, 78, 19-33. [CrossRef]

12. Zheng, J.; Dong, X.; Ji, J.; Su, H.; Ping Shum, P. Power-referenced refractometer with tilted fiber Bragg grating cascaded by chirped grating. Opt. Commun. 2014, 312, 106-109. [CrossRef]

13. Zhou, W.; Mandia, D.J.; Barry, S.T.; Albert, J. Absolute near-infrared refractometry with a calibrated tilted fiber Bragg grating. Opt. Lett. 2015, 40, 1713-1716. [CrossRef] [PubMed]

14. Wang, T.; Liu, K.; Jiang, J.; Xue, M.; Chang, P.; Liu, T. Temperature-insensitive refractive index sensor based on tilted moiré FBG with high resolution. Opt. Express 2017, 25, 14900-14909. [CrossRef] [PubMed]

15. Albert, J.; Shao, L.-Y.; Caucheteur, C. Tilted fiber Bragg grating sensors. Laser Photonics Rev. 2013, 7, 83-108. [CrossRef]

16. Chen, X.; Xu, J.; Zhang, X.; Guo, T.; Guan, B. Wide range refractive index measurement using a multi-angle tilted fiber Bragg grating. IEEE Photonics Technol. Lett. 2017, 29, 719-722. [CrossRef]

17. Jiang, B.; Bi, Z.; Wang, S.; Xi, T.; Zhou, K.; Zhang, L.; Zhao, J. Cascaded tilted fiber Bragg grating for enhanced refractive index sensing. Chin. Phys. B 2018, 27, 114220. [CrossRef] 
18. Gu, B.; Qi, W.; Zheng, J.; Zhou, Y.; Shum, P.P.; Luan, F. Simple and compact reflective refractometer based on tilted fiber Bragg grating inscribed in thin-core fiber. Opt. Lett. 2014, 39, 22-25. [CrossRef]

19. Guo, T.; González-Vila, Á.; Loyez, M.; Caucheteur, C. Plasmonic Optical Fiber-Grating Immunosensing: A Review. Sensors 2017, 17, 2732. [CrossRef]

20. González-Vila, Á.; Ioannou, A.; Loyez, M.; Debliquy, M.; Lahem, D.; Caucheteur, C. Surface plasmon resonance sensing in gaseous media with optical fiber gratings. Opt. Lett. 2018, 43, 2308-2311. [CrossRef] [PubMed]

21. Shen, C.; Zhong, C.; Liu, D.; Lian, X.; Zheng, J.; Wang, J.; Semenova, Y.; Farrell, G.; Albert, J.; Donegan, J.F. Measurements of milli-Newton surface tension forces with tilted fiber Bragg gratings. Opt. Lett. 2018, 43, 255-258. [CrossRef]

22. Baiad, M.D.; Gagné, M.; Madore, W.-J.; De Montigny, E.; Godbout, N.; Boudoux, C.; Kashyap, R. Surface plasmon resonance sensor interrogation with a double-clad fiber coupler and cladding modes excited by a tilted fiber Bragg grating. Opt. Lett. 2013, 38, 4911-4914. [CrossRef] [PubMed]

23. Hu, X.; Mégret, P.; Caucheteur, C. Surface plasmon excitation at near-infrared wavelengths in polymer optical fibers. Opt. Lett. 2015, 40, 3998-4001. [CrossRef] [PubMed]

24. Renoirt, J.-M.; Debliquy, M.; Albert, J.; Ianoul, A.; Caucheteur, C. Surface plasmon resonances in oriented silver nanowire coatings on optical fibers. J. Phys. Chem. C 2014, 118, 11035-11042. [CrossRef]

25. Jiang, B.; Lu, X.; Gan, X.; Qi, M.; Wang, Y.; Han, L.; Mao, D.; Zhang, W.; Ren, Z.; Zhao, J. Graphene-coated tilted fiber-Bragg grating for enhanced sensing in low-refractive-index region. Opt. Lett. 2015, 40, 3994-3997. [CrossRef] [PubMed]

26. Renoirt, J.-M.; Zhang, C.; Debliquy, M.; Olivier, M.-G.; Mégret, P.; Caucheteur, C. High-refractive-index transparent coatings enhance the optical fiber cladding modes refractometric sensitivity. Opt. Express 2013, 21, 29073-29082. [CrossRef] [PubMed]

27. Jiang, B.; Lu, X.; Mao, D.; Wang, Y.; Zhang, W.; Gan, X.; Zhao, J. Carbon nanotube-deposited tilted fiber Bragg grating for refractive index and temperature sensing. IEEE Photonics Technol. Lett. 2016, 28, $994-997$. [CrossRef]

28. Li, Z.; Yu, Z.; Yan, B.; Ruan, X.; Zhang, Y.; Dai, Y. Theoretical analysis of tuning property of the graphene integrated excessively tilted fiber grating for sensitivity enhancement. J. Opt. Soc. Am. B 2019, 36, 108-118. [CrossRef]

29. Zhou, K.; Zhang, L.; Chen, X.; Bennion, I. Optic sensors of high refractive-index responsivity and low thermal cross sensitivity that use fiber Bragg gratings of $>80^{\circ}$ tilted structures. Opt. Lett. 2006, 31, 1193-1195. [CrossRef] [PubMed]

30. Yan, Z.; Wang, H.; Wang, C.; Sun, Z.; Yin, G.; Zhou, K.; Wang, Y.; Zhao, W.; Zhang, L. Theoretical and experimental analysis of excessively tilted fiber gratings. Opt. Express 2016, 24, 12107-12115. [CrossRef]

31. Chen, X.; Zhou, K.; Zhang, L.; Bennion, I. In-fiber twist sensor based on a fiber Bragg grating with $81^{\circ}$ tilted structure. IEEE Photonics Technol. Lett. 2006, 18, 2596-2598. [CrossRef]

32. Yan, Z.; Sun, Z.; Zhou, K.; Luo, B.; Li, J.; Wang, H.; Wang, Y.; Zhao, W.; Zhang, L. Numerical and experimental analysis of sensitivity-enhanced RI sensor based on Ex-TFG in thin cladding fiber. J. Lightwave Technol. 2015, 33, 3023-3027. [CrossRef]

33. Mou, C.; Zhou, K.; Yan, Z.; Fu, H.; Zhang, L. Liquid level sensor based on an excessively tilted fibre grating. Opt. Commun. 2013, 305, 271-275. [CrossRef]

34. Luo, B.; Wu, S.; Zhang, Z.; Zou, W.; Shi, S.; Zhao, M.; Zhong, N.; Liu, Y.; Zou, X.; Wang, L.; et al. Human heart failure biomarker immunosensor based on excessively tilted fiber gratings. Biomed. Opt. Express 2017, 8, 57-67. [CrossRef]

35. Jiang, B.; Zhou, K.; Wang, C.; Sun, Q.; Yin, G.; Tai, Z.; Wilson, K.; Zhao, J.; Zhang, L. Label-free glucose biosensor based on enzymatic graphene oxide-functionalized tilted fiber grating. Sens. Actuators B Chem. 2018, 254, 1033-1039. [CrossRef]

36. Albert, J. Tilted fiber Bragg gratings as multi-sensors. Opt. Photonics News 2011, 22, 28-33. [CrossRef]

37. Zhou, K.; Zhang, L.; Chen, X.; Bennion, I. Low thermal sensitivity grating devices based on Ex- $45^{\circ}$ tilting structure capable of forward-propagating cladding modes coupling. J. Lightwave Technol. 2006, 24, 5087-5094. [CrossRef]

38. Jiang, B.; Yin, G.; Zhou, K.; Wang, C.; Gan, X.; Zhao, J.; Zhang, L. Graphene-induced unique polarization tuning properties of excessively tilted fiber grating. Opt. Lett. 2016, 41, 5450-5453. [CrossRef] [PubMed] 
39. Guo, T.; Liu, F.; Guan, B.-O.; Albert, J. Polarimetric multi-mode tilted fiber grating sensors. Opt. Express 2014, 22, 7330-7336. [CrossRef]

40. Chung, K.-W.; Yin, S. Analysis of a widely tunable long-period grating by use of an ultrathin cladding layer and higher-order cladding mode coupling. Opt. Lett. 2004, 29, 812-814. [CrossRef]

41. Yang, J.; Yang, L.; Xu, C.-Q.; Xu, C.; Huang, W.; Li, Y. Long-period grating refractive index sensor with a modified cladding structure for large operational range and high sensitivity. Appl. Opt. 2006, 45, 6142-6147. [CrossRef] [PubMed]

42. Bashkatov, A.N.; Genina, E.A. Water Refractive Index in Dependence on Temperature and Wavelength: A Simple Approximation; SPIE: Bellingham, WA, USA, 2003; Volume 5068.

43. Schiebener, P.; Straub, J.; Sengers, J.M.H.L.; Gallagher, J.S. Refractive index of water and steam as function of wavelength, temperature and density. J. Phys. Chem. Ref. Data 1990, 19, 677-717. [CrossRef]

44. Lide, D.R. (Ed.) Index of Refractive of Water in CRC Handbook of Chemistry and Physics, Internet Version; CRC Press: Boca Raton, FL, USA, 2005.

(C) 2019 by the authors. Licensee MDPI, Basel, Switzerland. This article is an open access article distributed under the terms and conditions of the Creative Commons Attribution (CC BY) license (http://creativecommons.org/licenses/by/4.0/). 